\title{
Hyperoxalemia Leads to Oxidative Stress in Endothelial Cells and Mice with Chronic Kidney Disease
}

\author{
Ke Sun ${ }^{a}$ Xiaojing Tang ${ }^{a}$ Shuwei Song ${ }^{a}$ Yuan Gao ${ }^{b}$ Hongjing Yu ${ }^{b}$ \\ Ningyun Sun ${ }^{b}$ Bin Wen ${ }^{b}$ Changlin $\mathrm{Mei}^{\mathrm{a}}$ \\ aKidney Institute, Department of Nephrology, Changzheng Hospital, Naval Medical University, Shanghai, China; \\ bPharmaceutical R\&D Center of SPH Sine Pharmaceutical, Laboratories Co., Ltd., Shanghai Engineering Research \\ Center of Innovative Probiotic Drugs, Shanghai, China
}

\section{Keywords}

End-stage renal disease $\cdot$ Hyperoxalemia $\cdot$ Vascular calcification - Oxidative stress

\begin{abstract}
Introduction: Cardiovascular disease is the most common cause of morbidity and mortality in patients with ESRD. In addition to phosphate overload, oxalate, a common uremic toxin, is also involved in vascular calcification in patients with ESRD. The present study investigated the role and mechanism of hyperoxalemia in vascular calcification in mice with uremia. Methods: A uremic atherosclerosis (UA) model was established by left renal excision and right renal electrocoagulation in $\mathrm{apoE}^{-/-}$mice to investigate the relationship between oxalate loading and vascular calcification. After 12 weeks, serum and vascular levels of oxalate, vascular calcification, inflammatory factors (TNF- $a$ and IL-6), oxidative stress markers (malondialdehyde [MDA], and advanced oxidation protein products [AOPP]) were assessed in UA mice. The oral oxalate-degrading microbe Oxalobacter formigenes ( $O$. formigenes) was used to evaluate the effect of a reduction in oxalate levels on vascular calcification. The mechanism underlying the effect of oxalate loading on vascular calcification was assessed in cultured human aortic en-
\end{abstract}

karger@karger.com www.karger.com/kbr

Karger"

BOPEN ACCESS
(C) 2021 The Author(s)

Published by S. Karger AG, Basel

This is an Open Access article licensed under the Creative Commons Attribution-NonCommercial-4.0 International License (CC BY-NC) (http://www.karger.com/Services/OpenAccessLicense), applicable to the online version of the article only. Usage and distribution for commercial purposes requires written permission. dothelial cells (HAECs) and human aortic smooth muscle cells (HASMCs). Results: Serum oxalate levels were significantly increased in UA mice. Compared to the control mice, UA mice developed more areas of aortic calcification and showed significant increases in aortic oxalate levels and serum levels of oxidative stress markers and inflammatory factors. The correlation analysis showed that serum oxalate levels were positively correlated with the vascular oxalate levels and serum MDA, AOPP, and TNF-a levels, and negatively correlated with superoxide dismutase activity. The $O$. formigenes intervention decreased serum and vascular oxalate levels, while did not improve vascular calcification significantly. In addition, systemic inflammation and oxidative stress were also improved in the $O$. formigenes group. In vitro, high concentrations of oxalate dose-dependently increased oxidative stress and inflammatory factor expression in HAECs, but not in HASMCs. Conclusions: Our results indicated that hyperoxalemia led to the systemic inflammation and the activation of oxidative stress. The reduction in oxalate levels by 0 . formigenes might be a promising treatment for the prevention of oxalate deposition in calcified areas of patients with ESRD.

(c) 2021 The Author(s)

Published by S. Karger AG, Basel

Co-first authors: Ke Sun and Xiaojing Tang.
Correspondence to:

Bin Wen, wenbin@sinepharm.com

Chang-Lin Mei, changlinmei@smmu.edu.cn 


\section{Introduction}

Cardiovascular disease (CVD) is the most common cause of morbidity and mortality in patients with CKD and ESRD [1]. Vascular calcification induced by hyperphosphatemia has been suggested to be a crucial factor in the pathophysiology of CVD [2]. However, it does not fully explain the extremely high rate of vascular calcification in patients with ESRD, and other factors that might be involved $[3,4]$. Uremic toxins produced by disturbances in the intestinal flora, including sulfuric acid, cresol, indole, and oxalate, were indicated to be associated with a high CVD incidence rate and high mortality rate in patients with ESRD $[5,6]$.

Oxalate is one of the small-molecule toxins retained in patients with ESRD. A positive linear correlation was observed between serum oxalate and creatinine levels in patients with ESRD, and oxalate is difficult to remove sufficiently by conventional hemodialysis $[7,8]$. The normal range of serum oxalate levels is approximately $1-5 \mu \mathrm{mol} / \mathrm{L}$, while serum oxalate levels in patients with ESRD are 4-78 times the normal limits, even as high as $200 \mu \mathrm{mol} / \mathrm{L}$ [9]. Gulhan et al. [10] reported that serum oxalate levels in hemodialysis patients are positively correlated with the pulse wave conduction velocity, central artery systolic blood pressure, and diastolic blood pressure, which were independent predictors of increased cardiovascular morbidity and mortality [10]. Furthermore, oxalate deposits were observed in atherosclerotic plaques during autopsies of patients with atherosclerosis [11]. In vivo, a uremic mouse model was established in C57BL/ 6 mice by providing a high oxalate diet for 3 weeks. The arterial pressure was significantly increased, accompanied by significant cardiac fibrosis [12]. In vitro, high oxalate levels increase the intracellular calcium concentration in endothelial cells [13] and inhibit the proliferation and remigration of endothelial cells [14], thus affecting the re-endothelialization of injured blood vessels and promoting the progression of arteriosclerosis. Based on these studies, oxalate overload might play an important role in vascular calcification in patients with ESRD.

Oxalobacterformigenes (O.formigenes) is an anaerobic bacterium that reduces serum and urinary oxalate levels by degrading oxalate in the intestinal tract [15-17]. Gulhan et al. [10] found that the lack of $O$. formigenes in hemodialysis patients was related to the increased serum oxalate level. Thus, increased colonization of $O$. formigenes might potentially decrease serum oxalate levels and improve vascular calcification in patients with ESRD. In this study, we investigated the role and mechanism of hy- peroxalemia in the development of vascular calcification in models of ESRD and the potential therapeutic effect of O. formigenes.

\section{Materials and Methods}

Mouse Model of Uremic Atherosclerosis

All animal studies were approved by the Ethics Committee of Naval Medical University. The mice were housed in polycarbonate cages under pathogen-free conditions on a 12-h light-dark cycle at the Animal Center of Naval Medical University (Shanghai, China) and provided free access to water and a high-fat diet for 3 months. The components of the high-fat diet as listed by the manufacturer (Jiangsu Synergetic Pharmaceutical Bioengineering Co., Ltd.) were a standard diet, $15 \%$ fat, $1 \%$ cholesterol, and $0.35 \%$ cholic acid.

Eight-week-old male apoE ${ }^{-/-}$mice (Kavens Experimental Animal Co., Ltd. Changzhou, China) were divided into 3 groups $(n=$ 15 mice per group): the control group, uremic atherosclerosis (UA) group, and UA group treated with $O$. formigenes intervention $(\mathrm{UA}+\mathrm{OxF})$. We used a 2 -step procedure to create a UA model. In brief, we applied cortical electrocautery to the right kidney through a $2-\mathrm{cm}$ flank incision and performed left total nephrectomy through a similar incision 2 weeks later, as described by Gagnon et al. [18]. Penicillin was administered for 3 days after the operation to prevent infection. In the control group, both kidneys were exposed during the 2 operations, and no surgical intervention was performed.

\section{Isolation and Purification of O. Formigenes from Fresh Human} Feces

First, the anaerobic environment was optimized, which would not hinder the growth of $O$. formigenes (the ratio of $\mathrm{N}_{2}, \mathrm{CO}_{2}$, and $\mathrm{H}_{2}$ in the anaerobic tank was 85:10:5). Second, a sterile cotton swab was dipped in fresh feces and diluted with normal saline to $10^{-3}$ and prepared the $\mathrm{OxD}$ agar solid medium and liquid $\mathrm{OxB}$ medium, as previously described $[19,20]$. Then, $100 \mu \mathrm{L}$ of diluent was added to the plate, and $5 \mathrm{~mL}$ of $\mathrm{OxD}$ agar solid medium was added at a temperature of $45^{\circ} \mathrm{C}$. The culture medium was quickly spread on the bottom of the plate with the coating rod. After the medium had cooled and solidified, the plate was cultured in an anaerobic tank at $37^{\circ} \mathrm{C}$. When some colonies on the plate were visible as 0.1 - to $0.3-\mathrm{mm}$ transparent dots or fusiform colonies, they were separated and purified. An inoculating loop was used to pick clear colonies on the $\mathrm{OxD}$ medium, and the bacteria were added to the liquid $\mathrm{OxB}$ medium and cultured on a shaker at $37^{\circ} \mathrm{C}$. After 2 days of culture, the presence of the following phenomena indicated that the bacteria grew well and produced gas: the culture medium changed from yellowish and clear to turbid with white granular suspended solids, white particles were observed on the inner wall of the vial, and the sealing pressure of the silicone rubber stopper increased. One milliliter of bacterial solution was used to measure the bacterial concentration, and $1 \mathrm{~mL}$ of blank $\mathrm{OxB}$ medium was used as a control. The concentration of the $O$. formigenes bacterial solution after $48 \mathrm{~h}$ of culture was $10^{9} \mathrm{cfu} / \mathrm{mL}$. The prepared strains were stored in a $-80^{\circ} \mathrm{C}$ freezer until further experiments. In the $\mathrm{UA}+\mathrm{OxF}$ group, each $\mathrm{UA}$ mouse was gavaged with $0.1 \mathrm{~mL}$ of $O$. formigenes suspension per day for 12 weeks. The 
Table 1. Primer sequences for RT-PCR

\begin{tabular}{lll}
\hline Gene & Primers $\left(5^{\prime}-3^{\prime}\right)$ & \\
\cline { 2 - 3 } & forward & reverse \\
\hline TNF-a & CTTTGGGATCATTGCCCTGTG & CGAAGTGGTGGTCTTGTTGCT \\
IL-6 & CGAATCTCCGACCACCACTAC & TGCACATAAGCCTCGTTATCC \\
CRP & AAGCCCAGGGTGAGGAAGAGT & CCCGCCAAGATAGATGGTGTTA \\
GAPDH & TTCTTGCCTCTTGTCTCTT & ATTTCTTCCATTCTGTCTT \\
\hline
\end{tabular}

other groups were gavaged with $0.1 \mathrm{~mL}$ of normal saline per day for 12 weeks as a control.

\section{Biochemical Analysis}

Under short-term anesthesia with diethyl ether (Meilunbio, China), blood samples were obtained from the inner canthal orbital vein. The samples were centrifuged at 4,000 rpm for $10 \mathrm{~min}$ at $4^{\circ} \mathrm{C}$, and serum was stored at $-80^{\circ} \mathrm{C}$ before further processing. Twenty-four-hour urine samples were collected in a tube containing $1 \mathrm{~mL}$ of mineral oil to prevent evaporation and $50 \mu \mathrm{L}$ of $2 \%$ sodium azide to prevent bacterial growth. Serum levels of urea nitrogen, high-density lipoprotein, low-density lipoprotein, total cholesterol, triglycerides, calcium, and phosphate were assessed using a Hitachi 917 autoanalyzer (Roche, France). Superoxide dismutase (SOD) activity and malondialdehyde (MDA) levels were measured according to the manufacturer's instructions (BioTNT, China). Serum levels of advanced oxidation protein products (AOPP) were measured using an OxiSelectAOPP Assay Kit (Cellbio, South Korea). TNF- $\alpha$ and IL- 6 levels were measured using ELISA (Anogen, China) according to the manufacturer's instructions. Serum and urine oxalate levels were detected using ion chromatography (Dionex IonPacTMAS14, $4 \times 250 \mathrm{~mm}$ ).

\section{Qualitative and Quantitative Evaluation of Aortic \\ Calcification \\ Qualitative Evaluation of Aortic Calcification}

The aortic arch and descending aorta were thoroughly cleaned of adventitial fat and cut open longitudinally. Vascular calcifications were evaluated by performing Von Kossa staining in cryosections of the aortic tissue. In brief, the cryosections were placed in a $1 \%$ silver nitrate solution (Sigma Aldrich) for $30 \mathrm{~min}$ in the dark. Then, they were fixed with a 5\% sodium thiosulfate solution for 1 min and stained with basic fuchsin. Calcium deposits appeared black on the bright red-colored surrounding tissue. Data are presented as the relative proportion of the calcified area to the total surface area of calcification lesions, as described previously [21].

Quantitative Evaluation of Vascular Oxalate and Phosphate

Levels

Aortic oxalate and phosphate levels were quantitatively detected using ion chromatography (Dionex IonPacTMAS14, $4 \times 250$ $\mathrm{mm})$. The blood vessels were shredded and placed in a centrifuge tube. After adding $5 \% \mathrm{HCl}$, the samples were extracted with ultrasound for $30 \mathrm{~min}$ and then centrifuged at 15,000 rpm for $10 \mathrm{~min}$ at $4^{\circ} \mathrm{C}$. The same volume of chloroform was added to the supernatant, and the mixture was centrifuged at $15,000 \mathrm{rpm}$ for $5 \mathrm{~min}$ at $4^{\circ} \mathrm{C}$. Linear dilutions of oxalate and phosphate solutions were prepared according to the instructions, and the contents of oxalate and phosphate in the vascular wall were calculated from a standard curve.

\section{Histology}

Paraffin-embedded mouse kidney sections ( $3 \mathrm{~mm}$ thickness) were prepared using a routine procedure. The sections were stained with periodic acid-Schiff staining reagents using a standard protocol described in a previous study [22].

\section{Cell Culture}

Primary human aortic endothelial cells (HAECs) and human aortic smooth muscle cells (HASMCs) were purchased from Science Cell Research Laboratories (Carlsbad, CA, USA) and cultured as previously described [23]. In brief, HAECs were grown in endothelial culture medium (No. 1001, Science Cell) containing $5 \% \mathrm{fe}$ tal bovine serum (No. 0025), 1\% endothelial cell growth supplement (No. 1052), and 1\% penicillin/streptomycin solution (No. $0503)$ at $37^{\circ} \mathrm{C}$ with $5 \% \mathrm{CO}_{2}$. All experiments were performed on HAECs between passages 2 and 5. HASMCs were cultured in smooth muscle cell medium (Cat. \#1101) supplemented with $2 \%$ fetal bovine serum, 1\% SMCGS, and 1\% penicillin and streptomycin. Experiments were performed using cells from passages 3-6. HAECs and HASMCs were treated with normal medium, 100 $\mu \mathrm{mol} / \mathrm{L}$ oxalate, $200 \mu \mathrm{mol} / \mathrm{L}$ oxalate, or and $500 \mu \mathrm{mol} / \mathrm{L}$ oxalate for $6 \mathrm{~h}$ or $24 \mathrm{~h}$. All other reagents were obtained from Sigma.

\section{MDA Levels and SOD Activity Assays}

Confluent HAECs and HASMCs cultured in 6-well plates were incubated with different doses of oxalate $(0,50,100,200$, or 500 $\mu \mathrm{mol})$ for $24 \mathrm{~h}$. Then, the cell supernatant was collected. SOD activity and MDA levels were measured according to the manufacturer's instructions (Bio TNT, China).

Measurement of Intracellular Reactive Oxygen Species Levels

For measurement of reactive oxygen species (ROS) production, HAECs and HASMCs cultured in 6-well plates were labeled for 30 min with $10 \mu \mathrm{M}$ of 6-carboxy-H2DCF-DA-di-AM. Cells were washed with PBS and treated for $6 \mathrm{~h}$ with sodium oxalate at different concentrations. After $6 \mathrm{~h}$, the production of ROS was measured by flow cytometry. The production of intracellular ROS was detected by measuring the fluorescence of 6-carboxy-H2DCF-DA-diAM by the SpectraMax i3x Fluorescence multi-well plate reader (USA) at excitation and emission wavelengths of 485 and $525 \mathrm{~nm}$, respectively, and normalized to cellular protein concentrations. 
Table 2. Serum and urinary biochemical parameters at week 12

\begin{tabular}{lccc}
\hline & $\begin{array}{l}\text { Control group } \\
(N=15)\end{array}$ & $\begin{array}{l}\mathrm{UA} \mathrm{group}^{\mathrm{a}} \\
(N=13)\end{array}$ & $\begin{array}{l}\mathrm{UA}+\mathrm{OxF}^{\mathrm{b}} \\
(N=13)\end{array}$ \\
\hline Body weight, g & $28.09 \pm 0.68$ & $25.84 \pm 0.57^{* *}$ & $25.81 \pm 0.94$ \\
Serum urea, mmol/L & $6.53 \pm 1.14$ & $22.66 \pm 6.34^{* *}$ & $21.32 \pm 4.05$ \\
TC, mmol/L & $21.11 \pm 4.25$ & $30.1 \pm 1.72^{* *}$ & $30.21 \pm 3.62$ \\
TG, $\mathrm{mmol} / \mathrm{L}$ & $1.21 \pm 0.34$ & $1.38 \pm 0.74$ & $1.25 \pm 0.46$ \\
$\mathrm{HDL}, \mathrm{mmol} / \mathrm{L}$ & $2.97 \pm 0.40$ & $2.68 \pm 0.69$ & $2.92 \pm 0.52$ \\
LDL, mmol/L & $19.32 \pm 4.93$ & $26.2 \pm 4.44^{* *}$ & $24.20 \pm 4.60$ \\
Serum calcium, mmol/L & $2.32 \pm 0.14$ & $2.33 \pm 0.13$ & $2.30 \pm 1.09$ \\
Serum phosphate, $\mathrm{mmol} / \mathrm{L}$ & $2.21 \pm 0.34$ & $3.80 \pm 0.95^{* *}$ & $3.46 \pm 1.09$ \\
Serum oxalate, $\mu \mathrm{mol} / \mathrm{L}$ & $7.22 \pm 1.54$ & $26.9 \pm 5.46^{* *}$ & $20.39 \pm 1.76^{*}$ \\
Urinary oxalate excretion, $\mu \mathrm{mol} / 24 \mathrm{~h}$ & $0.42 \pm 0.07$ & $0.23 \pm 0.06^{*}$ & $0.16 \pm 0.04^{*}$ \\
Urinary calcium, mg/24 h & $7.39 \pm 0.92$ & $13.89 \pm 1.62^{*}$ & $15.86 \pm 1.12^{*}$ \\
\hline
\end{tabular}

UA, uremic atherosclerosis. ${ }^{a}$ Compared to the control group. ${ }^{b}$ Compared to the UA group. $* p<0.05 .{ }^{* *} p<$ 0.001 .

\section{Real-Time Quantitative PCR}

Total RNA was extracted from HAECs and HASMCs by using RNAiso Plus (Takara). cDNA was synthesized by reverse transcriptase M-MLV (Takara). RT-qPCR was performed by using FastStart Universal SYBR Master (Roche) in an Applied Biosystems ViiA7 system (Thermo Fisher Scientific). RT-PCR primer sequences are listed in Table 1. GAPDH was used as the endogenous control. The relative amount of mRNA compared to the internal control was calculated using the $2^{-\Delta \Delta C T}$ method [24].

\section{Western Blot Analysis}

Protein samples were separated using $10 \%$ sodium dodecyl sulfate polyacrylamide gel electrophoresis ( $80 \mathrm{~g}$ of total protein per lane) and transferred onto a polyvinylidene fluoride membrane (Merck Millipore, Danver, MA, USA). In addition, gels stained with bromophenol blue were used to confirm the equal amounts of protein loaded on each lane. The membranes were incubated overnight at $4^{\circ} \mathrm{C}$ with rabbit anti-p-JAK2 (1:1,000, CST), rabbit anti-JAK2 (1:1,000, CST), rabbit anti-p-STAT3 (1:1,000, CST), or mouse anti-Stat3: mouse $(1: 1,000, \mathrm{CST})$. The membranes were extensively applied with PBS Tween and incubated for $2 \mathrm{~h}$ with horseradish peroxidase-conjugated secondary antibody $(1: 2,000$; Rockland)) at room temperature. The immune complexes were detected using a bicinchoninic acid protein assay kit (Pierce, Rockford, IL, USA). The density of the specific bands was analyzed using Image Lab 4 software (Bio-Rad, Hercules, CA, USA), and the expression levels of these proteins were normalized to GAPDH (1:10,000, Abcam).

\section{Statistical Analysis}

All data are presented as means \pm SD. Significant differences between means were analyzed using ANOVA followed by the Student-Newman-Keuls test. The Pearson correlation coefficient was calculated for a single factor correlation analysis, and $r$ was the correlation coefficient. A $p$ value $<0.05$ was considered statistically significant.

\section{Results}

\section{Oxalate Levels in Serum and Aortic Calcification}

Plaques Were Significantly Increased in the Mouse Model of UA

Mice were divided into the following 3 groups to determine the role of oxalate in the progression of UA: control, UA, and UA + OxF groups. Table 2 shows the findings of serum and urinary biochemical analyses conducted after 12 weeks. Urinary oxalate excretion in the UA group was significantly decreased compared with the control group (see online suppl. Fig. 1a; see www.karger. com/doi/10.1159/000516013 for all online suppl. material), and urinary calcium levels were increased in the UA groups (online suppl. Fig. 1b). In addition, significant increases in serum urea, total cholesterol, low-density lipoprotein, oxalate, and phosphate levels were observed in the UA group compared to the control group.

The histological evaluation of the aorta using Von Kossa staining revealed that aortic calcification was present in all 3 groups. The area of aortic calcification plaques was significantly increased in the UA group compared to the control group (Fig. 1). We further examined the oxalate level in aortic calcification plaques by performing ion chromatography to investigate the role of hyperoxalemia in aortic calcification (online suppl. Fig. 2). The vascular oxalate level in the UA group was significantly higher than that in the control group $(24.96 \pm 7.59 \mu \mathrm{g} / \mathrm{g}$ vs. $2.64 \pm 0.54 \mu \mathrm{g} / \mathrm{g}$, Fig. $2 \mathrm{a})$, reflecting the relatively high oxalate burden in the aortic plaques from the UA group. A positive correlation 
Fig. 1. Effects of oxalate on aortic calcification. A-C Representative photomicrographs of the aorta stained by the Von Kossa method in the control group (A), UA group (B), and $\mathrm{UA}+\mathrm{OxF}$ group (magnification $\times 40)(\mathbf{C})$; the area of aortic calcification in the 3 groups (D). UA, uremic atherosclerosis; UA + OxF group, UA group treated with $O$. formigenes intervention.

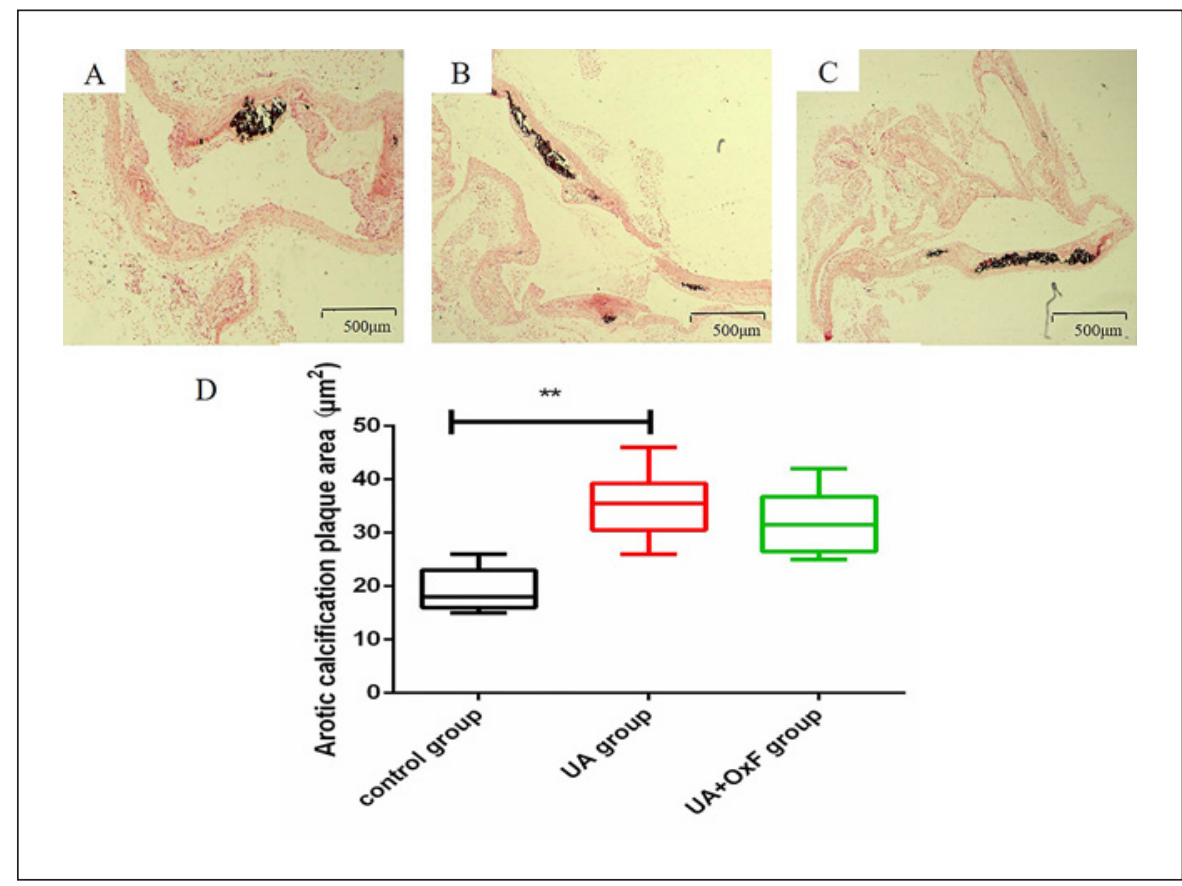

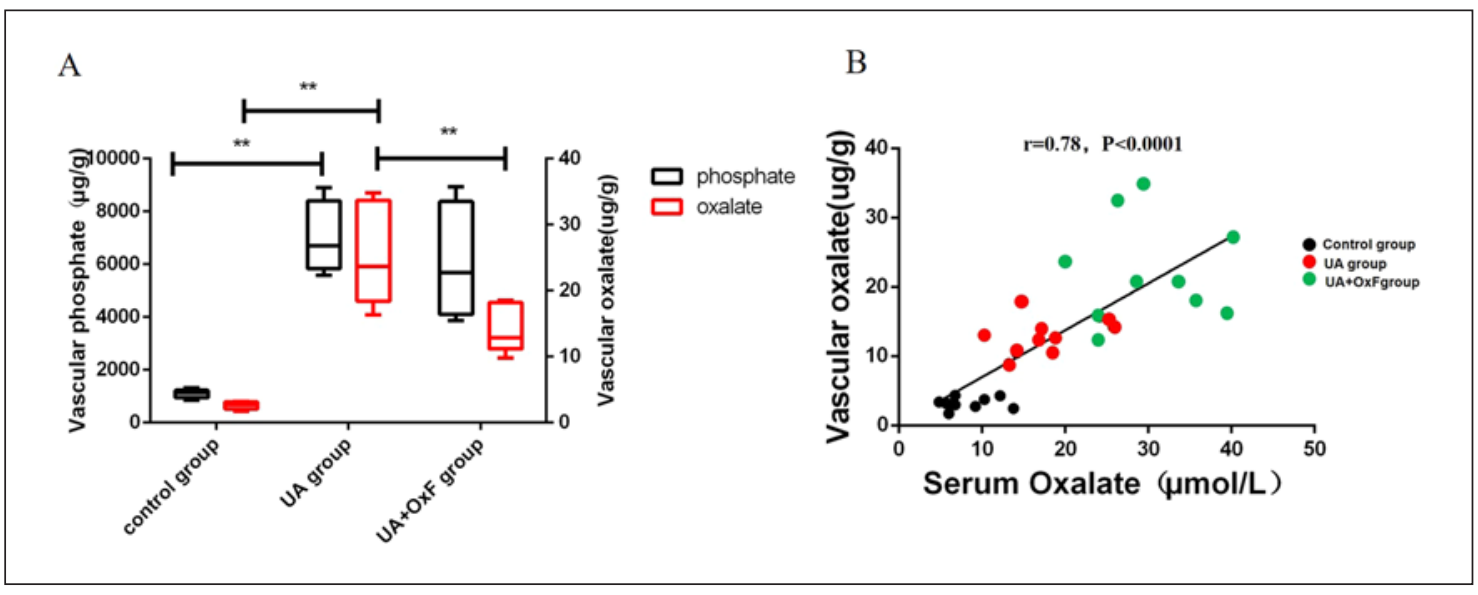

Fig. 2. Serum and vascular oxalate in 3 groups. A Vascular oxalate and phosphate in 3 groups. The unit of $\mu \mathrm{g} / \mathrm{g}$ represents the level of oxalate/phosphate per gram of blood vessels. B The correlation between serum oxalate and vascular oxalate levels $(r=0.78, p<0.001)$. All results were expressed as "means \pm SD." $N=10$ in each group. ${ }^{*} p<0.05 ;{ }^{* *} p<0.01$. UA, uremic atherosclerosis; UA + OxF group, UA group treated with $O$. formigenes intervention.

was identified between the vascular oxalate level and serum oxalate level (Fig. 2b).

After intervention with $O$. formigenes for 12 weeks, serum, urinary, and vascular oxalate levels all decreased significantly compared to xthe UA group (Table 2; Fig. 2a). $O$. formigenes was maintained at a concentration of $10^{6}$ $\mathrm{cfu} / \mathrm{g}$ in feces from the UA + OxF group, while no O. formigenes was detected in the UA and control groups (online suppl. Fig. 1c), indicating the considerable colonization of $O$. formigenes in the $\mathrm{UA}+\mathrm{OxF}$ group. However, the vascular calcification area was not decreased significantly after the O. formigenes intervention (Fig. 1). 


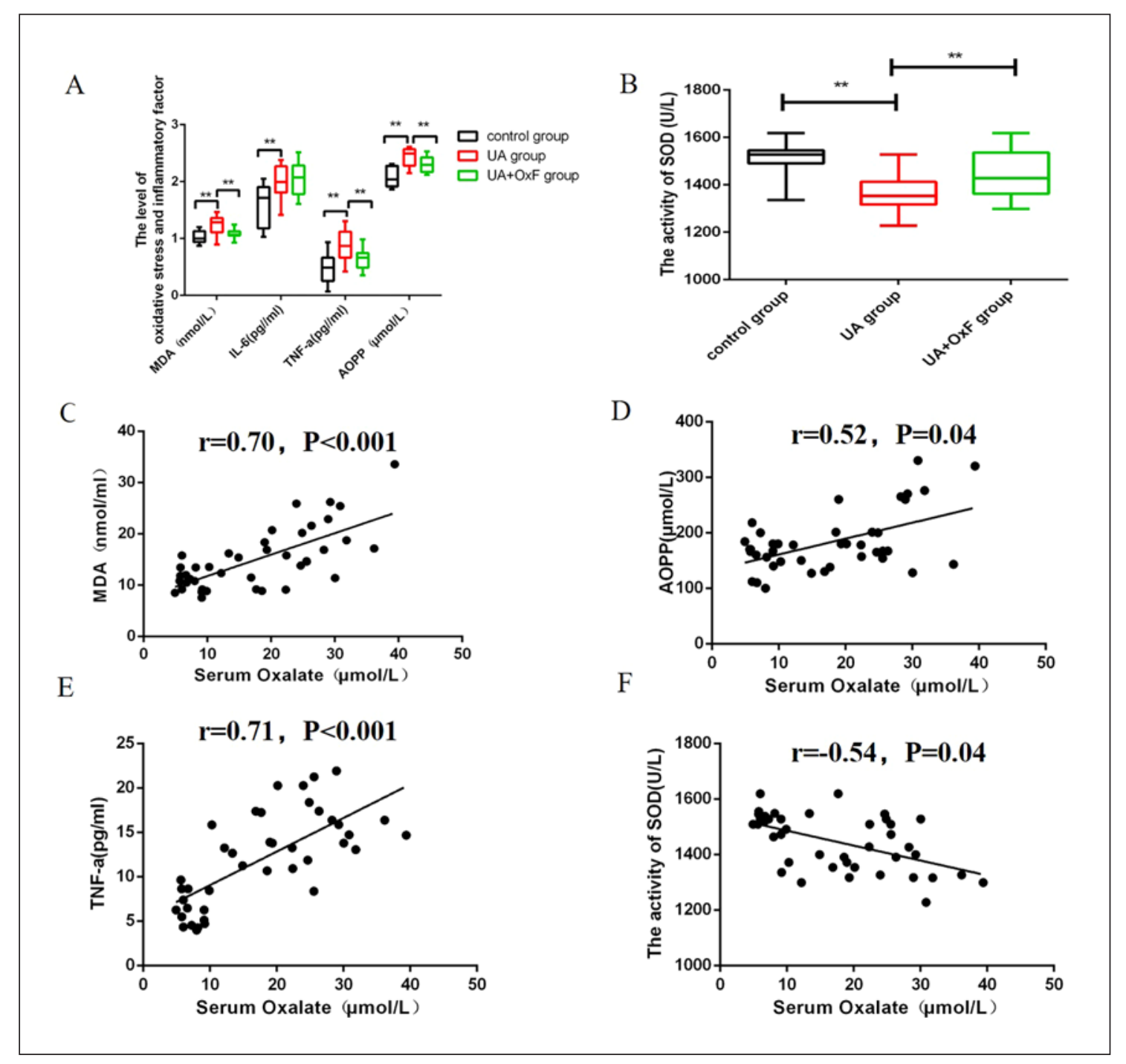

Fig. 3. Effects of hyperoxalemia on the oxidative stress markers and inflammatory factors expression. A, B Level of serum MDA, IL-6, AOPP, TNF- $\alpha(\mathbf{A})$, and SOD activity (B) among 3 groups. Results were expressed as "means \pm SD." $n=10$ in each group. C-F Correlation between serum oxalate and serum MDA levels $(r=0.70, p<0.001)$ (C), AOPP level $(r=0.52, p=0.004)(\mathbf{D})$, TNF- $\alpha$ level $(r=0.71, p<0.001)(\mathbf{E})$, and SOD activity $(\mathbf{F})(r=-0.54$, $p=0.004)$. UA, uremic atherosclerosis; MDA, malondialdehyde; AOPP, advanced oxidation protein products; SOD, superoxide dismutase; UA + OxF group, UA group treated with $O$. formigenes intervention.

Hyperoxalemia Induced Oxidative Stress and Inflammatory Gene Expression in the Mouse UA Model

Serum MDA, IL-6, AOPP, TNF- $\alpha$, and SOD levels were measured to further investigate the level of oxidative stress and the expression of inflammatory factors induced by hyperoxalemia. Compared to the control group, serum MDA, IL-6, AOPP, and TNF- $\alpha$ levels were significantly increased in the UA group, while SOD activity was sig- nificantly decreased ( $p<0.001$, Fig. 3a, b). In addition, serum oxalate levels positively correlated with serum MDA, AOPP, and TNF- $\alpha$ levels and negatively correlated with SOD activity ( $p<0.05$, Fig. $3 c-f)$. The administration of $10^{8} \mathrm{cfu}$ of $O$. formigenes significantly reduced serum MDA, AOPP, and TNF- $\alpha$ levels and increased serum SOD activity $(p<0.05$, Fig. 3a, b). However, significant differences in IL-6 levels were not observed between the $\mathrm{UA}$ group and $\mathrm{UA}+\mathrm{OxF}$ group. 


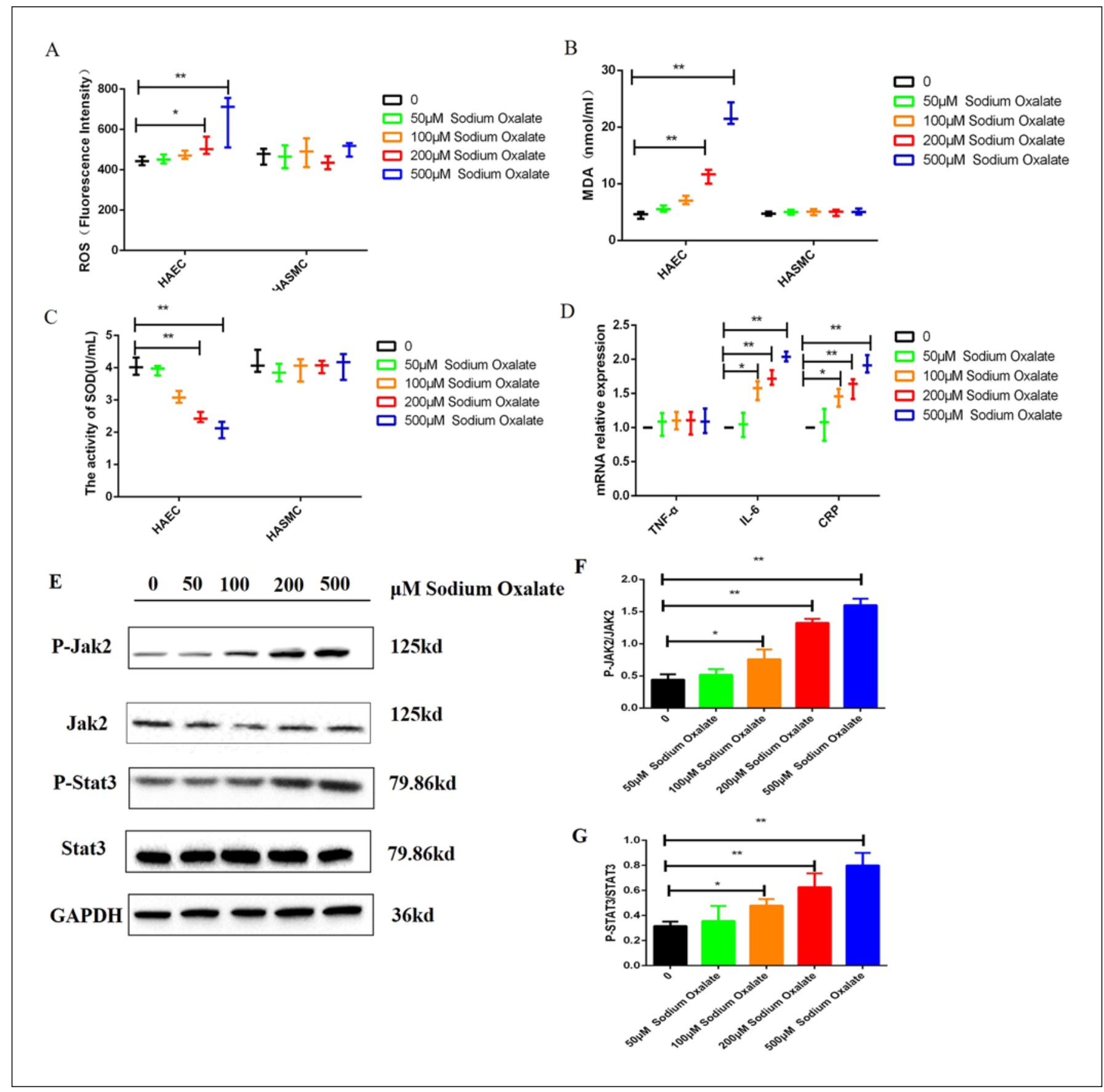

Fig. 4. High oxalate-induced oxidative stress and inflammatory factors expression in HAECs via the JAK2/STAT3 signaling pathway. A-C Expression of oxidative stress markers including ROS (A), MDA (B), and SOD (C)in HAECs and HASMCs stimulated by different levels of sodium oxalate. $\mathbf{D}$ mRNA expressions of inflammatory factors including TNF- $\alpha$, CRP, and IL-6 in HAECs. E-G High oxalate-induced JAK2 and STAT3 phosphorylation and activation in HAECs. Representative Western blots (E) and quantitative data $(\mathbf{F}, \mathbf{G})$ of phosphorylated JAK2 and STAT3 in HAECs stimulated by different levels of sodium oxalate. All results were expressed as "means \pm SD." $N=3$ in each group. * $p<0.05$; ${ }^{* *} p<$ 0.01 . HAEC, human aortic endothelial cells; HASMC, human aortic smooth muscle cells; MDA, malondialdehyde; SOD, superoxide dismutase; ROS, reactive oxygen species. 
Oxalate Overload Induced Oxidative Stress and

Inflammatory Factor Expression in HAECs via the JAK2/STAT3 Signaling Pathway

An in vitro experiment using cultured HAECs and HASMCs was performed to determine the target cells involved in oxalate-induced vascular calcification. Intracellular ROS generation, as measured by the average fluorescence intensity, was significantly increased in HAECs after 6 hof stimulation with 200 and $500 \mu \mathrm{M}$ sodium oxalate, while no change was observed in HASMCs (Fig. 4a). Similarly, oxalate caused a dose-dependent increase in the mRNA levels of MDA, IL-6, and CRP but led to a decrease in SOD activity in HAECs after $24 \mathrm{~h}$ of stimulation (Fig. 4b-d). The oxalate load did not alter the expression of MDA, IL-6, TNF- $\alpha$, or CRP in HASMCs (online suppl. Table 1).

HAECs were incubated with different concentrations of oxalate for $24 \mathrm{~h}$ to investigate the potential role of JAK2/STAT3 signaling in the activation of oxidative stress and inflammatory factor expression by hyperoxalemia in HAECs. Levels of the phosphorylated JAK2 and STAT3 proteins were analyzed using Western blotting. As shown in Fig. 4e, p-JAK2 and p-STAT3 levels were significantly upregulated in a concentration-dependent manner, suggesting the activation of the JAK2/STAT3 signaling pathway in response to high oxalate-induced HAEC injury.

\section{Discussion}

CKD has become a global health problem because of its high incidence and mortality rates. CVD is the most common cause of death in patients with ESRD, especially in patients on dialysis [25]. Evidence has revealed the harmful effects of phosphate overload on the cardiovascular system in patients with ESRD. However, phosphate binders are unable to fully reverse vascular calcification in patients with ESRD. The role of oxalate in the formation of calcium oxalate stones has been widely studied $[26,27]$. As an exogenous uremic toxin, serum oxalate was shown to positively correlate with the serum creatinine level, pulse wave conduction velocity, central artery systolic blood pressure, and diastolic blood pressure [18, 28]. In the present study, we investigated the role of oxalate in the formation of vascular calcification in uremia models.

Serum oxalate levels were significantly increased and urinary oxalate levels were significantly decreased in the UA group as kidney function deteriorated. In addition, the present study illustrated that oxalate was deposited on calcified vessels (in both the UA group and control group), and serum oxalate levels positively correlated with vascular oxalate levels. These findings are consistent with the results from the study by Nishizawa et al. [29] who showed that calcium oxalate might be one of the components of coronary artery calcification in dialysis patients. Furthermore, a significantly higher ratio of aortic oxalate to phosphate levels was observed in the UA group than in the control group, indicating that oxalate might play an important role in the development of vascular calcification in individuals with ESRD.

High oxalate concentrations have been shown to increase the production of reactive oxygen species in renal tubular epithelial cells, leading to endothelial injury and apoptosis, which plays an important role in the formation of kidney crystals $[14,30]$. Previous studies found that oxalate treatment increased the levels of $\mathrm{LDH}, \mathrm{H}_{2} \mathrm{O}_{2}$, MDA, and ROS in HK-2 cells, which indicated the activation of oxidative stress [31-33]. Consistent with the findings of urolithiasis studies, we found that oxidative stress was activated in uremic atherosclerotic mice with hyperoxalemia as well. Serum oxalate levels positively correlated with serum MDA, AOPP, and TNF- $\alpha$ levels and negatively correlated with SOD activity. In vitro, oxalate caused a dose-dependent increase in the secretion of inflammatory factors, including MDA, IL-6, TNF- $\alpha$, and $\mathrm{CRP}$, in HAECs but not in HASMCs, indicating that vascular endothelial cells might be the target cells of oxalateinduced vascular calcification. The JAK2/STAT3 signaling pathway plays an important role in regulating the secretion of inflammatory factors [34, 35]. Our study indicated a potential role for JAK2/STAT3 signaling in increasing the inflammatory factors and activating oxidative stress by high oxalate concentrations as p-JAK2 and p-STAT3 levels were significantly upregulated in HAECs. In studies of renal tubular cells, oxidative damage caused by high oxalate levels promotes the epithelial-mesenchymal transition (EMT). Given the tight association between oxalate loading and oxidative stress and the correlation between ROS levels and the EMT, the oxalate-induced EMT in vascular epithelial cells might be considered the potential mechanism of vascular calcification in patients with ESRD. Interestingly, our study did not observe a significant effect of high oxalate concentrations on HASMCs, which was probably related to the lack of the oxalate transporter SLC26 in HASMCs [36].

The $O$. formigenes intervention significantly decreased serum and urine oxalate levels, which was proven to be effective in reducing the risk of calcium oxalate kidney stones [37]. O. formigenes decreased both exogenous and 
endogenous oxalate levels. Based on our results, the expression of $O$. formigenes in feces was determined by qPCR (online suppl. Fig. 3), and O. formigenes effectively reduced the oxalate overload in uremic mice. After the $O$. formigenes intervention, the serum oxalate levels and oxalate levels in aortic calcification plaques were significantly decreased. Moreover, the administration of O. formigenes significantly reduced serum MDA, AOPP, and TNF- $\alpha$ levels and led to an increase in serum SOD activity, indicating that $O$. formigenes ameliorated the oxidative stress caused by hyperoxalemia.

The present study has some limitations. First, our study failed to verify a significant therapeutic effect of the $O$. formigenes intervention on the overall area of calcified plaques and glomerulosclerosis (online suppl. Fig. 4). The uremic mouse model was established for only 3 months. Due to the relatively short course of uremia, serum oxalate levels in this uremic mouse model were not as high as the levels we usually observe in uremic patients. This discrepancy might contribute to the result that vascular oxalate levels were not very high and the ratio of oxalate to phosphate levels was relatively low. Thus, we observed a trend of a decrease in the plaque area in the $\mathrm{UA}+\mathrm{OxF}$ group, which unfortunately did not reach statistical significance. Nevertheless, O. formigenes significantly reduced the vascular oxalate level and the serum inflammatory factor levels and improved oxidative stress. Second, we did not measure the atherosclerotic plaque by Oil Red O staining in the present study. Since high-fat diet could also induce atherosclerotic plaques in the $\mathrm{apoE}^{-/-}$mice, it would be also interesting to see if atherosclerotic plaque burden can be modified by $O$. formigenes treatment. Third, we observed associations between oxalate overload and oxidative stress and inflammation but did not further verify the putative cause-effect relationship by directly intervening in the inflammatory process related to oxalate loading. We did not further explore the mechanism of oxalate-induced vascular endothelial injury to determine whether it is mediated by oxalate transporters or the deposition of calcium oxalate crystals in the vascular wall. However, after considering these limitations, we propose that oxalate overload induces inflammation, oxidative stress, and vascular calcification in subjects with ESRD, and the present study provides insights into another important harmful effect of oxalate overload on patients with ESRD.

Our study is the first to suggest an important role for hyperoxalemia in vascular calcification in ESRD by inducing oxidative stress and inflammatory gene expression, leading to oxalate deposition in calcified areas. The administration of $O$. formigenes may reduce the serum oxalate level and improve the inflammatory state of patients with ESRD, which is of great clinical significance.

\section{Statement of Ethics}

The animal study was reviewed and approved by the Shanghai Changzheng Hospital's Biomedical Research Ethics Committee.

\section{Conflict of Interest Statement}

There are no conflicts of interest to declare. The results presented in this article have not been published previously.

\section{Funding Sources}

This work was granted by the National Key Research and Development Program of China (2016YFC0901502) and the National Natural Science Foundation of China $(81873595,81670612)$.

\section{Author Contributions}

Bin Wen and Changlin Mei designed the research; Ke Sun, Xiaojing Tang, and Changlin Mei analyzed the data; Ke Sun, Shuwei Song, Yuan Gao, Hongjing Yu, and Ningyun Sun performed the research; and Ke Sun, Xiaojing Tang, Bin Wen, and C. Mei wrote the manuscript.

\section{References}

1 Foley RN, Parfrey PS, Sarnak MJ. Clinical epidemiology of cardiovascular disease in chronic renal disease. Am J Kidney Dis. 1998; 32(3):S112-9.

2 Locatelli F, Bommer J, London GM, MartínMalo A, Wanner C, Yaqoob M, et al. Cardiovascular disease determinants in chronic renal failure: clinical approach and treatment. Nephrol Dial Transplant. 2001;16(8):459-68.
3 Longenecker JC, Coresh J, Powe NR. Traditional cardiovascular disease risk factors in dialysis patients compared with the general population: the choice study. J Am Soc Nephrol. 2002;13(5):1918-27.

4 Stack AG, Bloembergen WE. Prevalence and clinical correlates of coronary artery disease among new dialysis patients in the United States: a crosssectional study. J Am Soc Nephrol. 2001;12(3):1516-23.
5 Meijers BK, Evenepoel P. The gut-kidney axis: indoxyl sulfate, $\mathrm{p}$-cresyl sulfate and CKD progression. Nephrol Dial Transplant. 2011; 26(15):759-61.

6 Meijers B, Toussaint ND, Meyer T, Bammens B, Verbeke K, Vanrenterghem Y, et al. Reduction in protein-bound solutes unacceptable as marker of dialysis efficacy during alternatenight nocturnal hemodialysis. Am J Nephrol. 2011;34(11):226-32. 
7 Mydlik M, Derzsiova K. Oxalic acid: important uremic toxin (in Slovak). Vnitr Lek. 2010; 56(13):695-701.

8 France NC, Holland PT, Wallace MR. Contribution of dialysis to endogenous oxalate production in patients with chronic renal failure. Clin Chem. 1994;40(17):1544-8.

9 Tomson CR, Channon SM, Ward MK, Laker MF. Plasma oxalate concentration, oxalate clearance and cardiac function in patients receiving haemodialysis. Nephrol Dial Transplant. 1989;4(14):792-9.

10 Gulhan B, Turkmen K, Aydin M, Gunay M, Cikman A, Kara M. The relationship between serum oxalic acid, central hemodynamic parameters and colonization by oxalobacter formigenes in hemodialysis patients. Cardiorenal Med. 2015;5(13):164-74.

11 Fishbein GA, Micheletti RG, Currier JS, Currier JS, Singer E, Fishbein MC. Atherosclerotic oxalosis in coronary arteries. Cardiovasc Pathol. 2008;17(11):117-23.

12 Mulay SR, Eberhard JN, Pfann V, Marschner JA, Darisipudi MN, Daniel C, et al. Oxalateinduced chronic kidney disease with its uremic and cardiovascular complications in C57BL/6 mice. Am J Physiol Renal Physiol. 2016;310(8):F785-95.

13 Recht PA, Tepedino GJ, Siecke NW, Buckley MT, Mandeville JT, Maxfield FR, et al. Oxalic acid alters intracellular calcium in endothelial cells. Atherosclerosis. 2004;173(11):321-8.

14 Levin RI, Kantoff PW, Jaffe EA. Uremic levels of oxalic acid suppress replication and migration of human endothelial cells. Arteriosclerosis. 1990;10(14):198-207.

15 Hoppe B, Groothoff JW, Hulton SA, Cochat P, Niaudet P, Kemper MJ, et al. Efficacy and safety of oxalobacter formigenes to reduce urinary oxalate in primary hyperoxaluria. Nephrol Dial Transplant. 2011 Apr;26(11): 3609-15.

16 Hatch M, Cornelius J, Allison M, Sidhu H, Peck A, Freel RW. Oxalobacter sp. reduces urinary oxalate excretion by promoting enteric oxalate secretion. Kidney Int. 2006; 69(4):691-8.
17 Lieske JC. Probiotics for prevention of urinary stones. Ann Transl Med. 2017;5(4):29-8.

18 Gagnon RF, Gallimore B. Characterization of a mouse model of chronic uremia. Urol Res. 1988;16(4):119-26.

19 Dawson KA, Allison MJ, Hartman PA. Isolation and some characteristics of anaerobic oxalate-degrading bacteria from the rumen. Appl Environ Microbiol. 1980;40(4):833-9.

20 Zhang D, Tang X, Gao Y, Yuan Y, Yu H, Wen $\mathrm{B}$, et al. Role of oxalobacter fonnigenes in preventing calcium oxalate kidney stones. Chin J Nephrol. 2019;35(4):288-94.

21 Angulo J, Nguyen-Khoa T, Massy ZA, Drueke T, Serra J. Morphological quantification of aortic calcification from low magnification images. Image Anal Stereol. 2003;22(7):81-9.

22 Yang Y, Chen M, Zhou J, Lv J, Song S, Mei C, et al. Interactions between macrophages and cyst-lining epithelial cells promote kidney cyst growth in pkd1-deficient mice. J Am Soc Nephrol. 2018;29(9):2310-25.

23 Liu D, Huo Y, Chen S, Xu D, Yang B, Xue C, et al. Identification of key genes and candidated pathways in human autosomal dominant polycystic kidney disease by bioinformatics analysis. Kidney Blood Press Res. 2019;44(4):533-52.

24 Kageyama S, Yokoo H, Tomita K, KageyamaYahara N, Uchimido R, Matsuda N, et al. High glucose-induced apoptosis in human coronary artery endothelial cells involves upregulation of death receptors. Cardiovasc Diabetol. 2011;10(17):73-80.

25 Coresh J. Update on the burden of CKD. J Am Soc Nephrol. 2017;28(4):1020-2.

26 Whittamore JM, Hatch M. The role of intestinal oxalate transport in hyperoxaluria and the formation of kidney stones in animals and man. Urolithiasis. 2017;45(1):89-108.

27 Miller AW, Choy D, Penniston KL, Lange D. Inhibition of urinary stone disease by a multispecies bacterial network ensures healthy oxalate homeostasis. Kidney Int. 2019;96(1): $180-8$.

28 Selistre LDS, Cochat P, Rech DL, Parant F, de Souza VC, Dubourg L. Association between glomerular filtration rate (measured by highperformance liquid chromatography with iohexol) and plasma oxalate. J Bras Nefrol. 2018;40(1):73-6.
29 Nishizawa Y, Higuchi C, Nakaoka T, Omori $\mathrm{H}$, Ogawa T, Sakura H, et al. Compositional analysis of coronary artery calcification in dialysis patients in vivo by dual-energy computed tomography angiography. Ther Apher Dial. 2018;22(4):365-70.

30 Goncalves S, Pecoits-Filho R, Perreto S, Barberato SH, Stinghen AEM, Lima EGA, et al. Associations between renal function, volume status and endotoxaemia in chronic kidney disease patients. Nephrol Dial Transplant. 2006;21(10):2788-94.

31 Li D, Zhang D, Tang B, Zhou Y, Guo W, Kang $\mathrm{Q}$, et al. Exosomes from human umbilical cord mesenchymal stem cells reduce damage from oxidative stress and the epithelial-mesenchymal transition in renal epithelial cells exposed to oxalate and calcium oxalate monohydrate. Stem Cells Int. 2019;2019(5): 6935806-34.

32 Joshi S, Khan SR. Opportunities for future therapeutic interventions for hyperoxaluria: targeting oxidative stress. Expert Opin Ther Targets. 2019;23(5):379-91.

33 Liu H, Sun X-Y, Wang F-X, Ouyang J-M. Regulation on calcium oxalate crystallization and protection on HK-2 cells of tea polysaccharides with different molecular weights. Oxid Med Cell Longev. 2020;2020(15):5057123-6.

34 Xia Y, Xia H, Chen D, Liao Z, Yan Y. Mechanisms of autophagy and apoptosis mediated by JAK2 signaling pathway after spinal cord injury of rats. Exp Ther Med. 2017;14(2): 1589-93.

35 Xie J, Li Y, Dai J, He Y, Sun D, Dai C, et al. Olfactory ensheathing cells grafted into the retina of RCS rats suppress inflammation by down-regulating the JAK/STAT pathway. Front Cell Neurosci. 2019;13(13):341-7.

36 Alper SL, Sharma AK. The SLC26 gene family of anion transporters and channels. Mol Aspects Med. 2013 Apr-Jun;34(2-3):494-515.

37 Kaufman DW, Kelly JP, Curhan GC, Anderson TE, Dretler SP, Glenn M, et al. Oxalobacter formigenes may reduce the risk of calcium oxalate kidney stones. J Am Soc Nephrol. 2008;19(6):1197-203. 